\title{
Reduction of Backscattered Radiation in an X-Ray Room by Using Fabricated Iron Steel Grid
}

\author{
Abdullah Taher Qaed Naji, Mohamad Suhaimi Jaafar \\ School of Physics, Universiti Sains Malaysia, Pulau Pinang, Malaysia \\ Email: ataher8383@yahoo.com,msj@usm.my
}

Received May 16, 2013; revised June 3, 2013; accepted June 15, 2013

Copyright (c) 2013 Abdullah Taher Qaed Naji, Mohamad Suhaimi Jaafar. This is an open access article distributed under the Creative Commons Attribution License, which permits unrestricted use, distribution, and reproduction in any medium, provided the original work is properly cited.

\begin{abstract}
This study aims to improve a way for reducing backscattered radiation in an X-ray room. An iron steel grid, capable to absorb a significant portion of the backscattered radiation, was used. X-ray machine as a source for radiation was directed normally on the phantom, and the backscattered radiation was measured by using ion chamber. The measurements were recorded at various applied voltages (60 kvp to $120 \mathrm{kvp}$ ) and the fabricated grid was designed from iron steel constructed of perpendicular parallel strips mounted on a base. The results indicated that the use of iron steel grid was very effective in the reduction of backscattered radiation in an X-ray room up to about $46 \%$ by using fabricated iron steel grid.
\end{abstract}

Keywords: Reduction; Backscattered Radiation; X-Ray; Iron Steel; Grid

\section{Introduction}

The use of X-ray has extended and become very important in both diagnostic radiology imaging and radiotherapy. Nowadays, radiology seems to be a necessary procedure in diagnosis and treatment of patients. X-ray examinations have several clinical advantages. In a lot of ways it has preserved its priority over other diagnostic methods. In most cases, X-ray is the first choices of examination of the diagnostic algorithm. In fact, X-ray examination is still the most frequently used modality [1].

Scattered photons are radiations that change in direction as a result of interaction with some medium. These scattered photons are detrimental to contrast the image, reduce accurate representations of human anatomy, and increase the patients and workers dose [2,3].

There are two types: scattered radiation, and backscattered radiation. The side scattered radiation occurs when the photon scatters to the side or is reflected 90 degree from the initial photon beam. The back scattered radiation is, when the photon scatters is in a backward direction from the initial primary beam, at either 180 degree or at various angles [4].

Backscattered radiation contributes with a large portion of scattered radiation which is the main source of exposure to radiation workers in an X-ray room [5]. The physical characteristic of X-rays is scattered by the pa- tient and reaching the imaging detect on verification image quality. X-ray scatter is a complex phenomenon that can cause significant degradation of quality, primarily in term of contrast and contribute to image noise. In addition, it has important effects on the patients and workers in an X-ray room. That is to say, the scattered radiation not only reduces the image quality, but also gives additional unwanted dose to the patients and workers in an Xray room [6,7].

\section{Equipments and Methodology}

The equipments that were used in this experiment include an X-ray machine, ion chamber, and fabricated iron steel grid.

\subsection{X-Ray Machine}

The main component of $\mathrm{X}$-ray machine is $\mathrm{X}$-ray generator. The major components of an $\mathrm{X}$-ray generator are the tube, the high voltage generator, the control console, and the cooling system.

\subsection{Ion Chamber}

The ion chamber used for the measurement of backscattered X-ray photons was model Exradin A10. It is used with electrometer to measure dose, charge, and current. 


\subsection{Electrometer Max 4000}

The Ion Chamber was used with a Dosimeter Model MAX 4000 that automatically detects the start and the stop of radiation exposure by measuring the current crossing predetermined limit thresholds.

\subsection{Iron Steel Grid}

A scattered radiation grid consists of a system of vertical steel iron lamellae arranged at specific distances from each other as it is shown in Figure 1.

\section{Procedures}

For studying the effectiveness of iron steel grid on reduction of backscattered radiation, we have measured the backscattered dose at different field sizes. We fixed the detector at an angle of backscattering $160^{\circ}$ with the incident X-ray beam to measure backscattered dose only. And we used an applied voltage ranged (60 to $120 \mathrm{kvp}$ ) at one meter distance between X-ray machine source and target material (phantom) by using the grid that placed under target material, and without using the grid. Also, for comparing back scattered dose with different strip spacing, we have used different strip spacing ranged from 1 to $5 \mathrm{~cm}$.

\section{Results and Discussion}

The backscattered doses at different setups were plotted against the kvp to compare the effect of Iron Steel Grid on reduction backscattered radiation dose; Figure 2 shows the results of backscattered radiation dose measurements. It also shows a comparison between using grid and without using grid under the radiographic phantom.

Figure 2 shows the difference in the backscattered radiation dose between measuring dose with and without using the iron steel grid. It also shows the ability of grid to reduce backscattered radiation. In all measurements, it can be clearly seen the dose of X-ray is proportional to

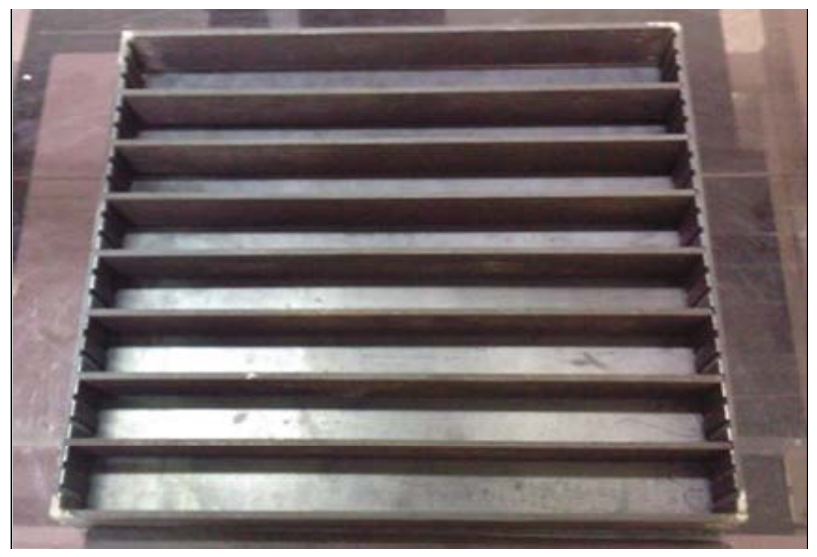

Figure 1. Iron steel grid $40 \times 40 \mathrm{~cm}^{2}$.

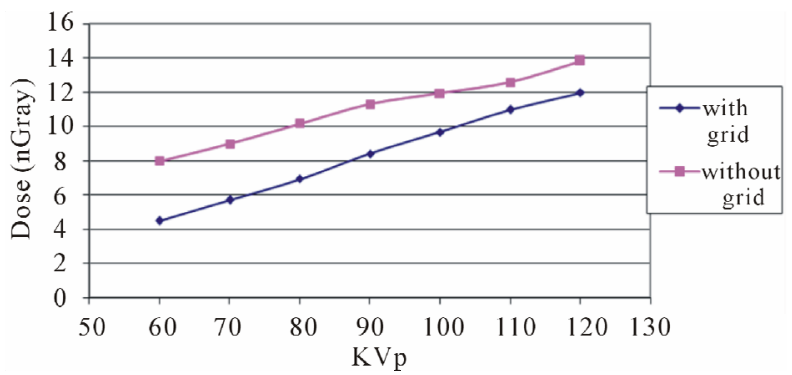

Figure 2. Backscattered radiation dose of $X$-ray at field size $10 \times 10 \mathrm{~cm}^{2}$.

the applied voltage (kvp) due to increase in the kvp the number of generated photons increase. This means that the energy and their maximal energy lead to increase the number of backscattered photons which reached to the detector.

On the other hand, to measure the effectiveness of grid in reduction of backscattered radiation dose, the percentage of reduction in backscattered radiation dose has calculated at an angle 160 degree with incident beam for 0.1 sec exposure time, at field size $10 \times 10 \mathrm{~cm}^{2}$, and current $400 \mathrm{~mA}$, as it is shown in Figure 3.

From Figure 3, the effectiveness of iron steel grid in reduction of backscattered radiation dose was observed to be more significant particularly at low applied voltage and the reduction of backscattered radiation up to about $46 \%$ by using iron steel grid. In addition, it shows the inversely proportion between the reduction of backscattered radiation and applied voltage of incident X-ray. The higher percentage of backscattered reduction is at low kvp than high kvp. The applied voltage leads to an increase in the energy of photon and an increase in the quantity of backscattered photons that reach to detector (Ion Chamber).

Different strip spacing ranged from 1 to $5 \mathrm{~cm}$ has been used at different applied voltages. Figure 4 shows the results of backscattered radiation do seat different spacing between the strips of grid.

From Figure 4, one can see the effect of increasing strip interspaces on reduction of backscattered radiation can be observed. The reduction of back-scattered radiation increases with reducing spacing between strips. The reduction is clearly high at smaller spacing between strips since the ability of strips of grid to trap the backscattered radiation when it hits the base of grid and wall of strips. So the ratio of trapped scattered photons will be increased by reducing the space between strips. In addition, it is useful to decrease the thickness of strips due to the backscattered radiation is a result of hitting surface lamellae (strip) by incident radiation which will backscatter in all directions.

\section{Conclusions}

The results show the effectiveness of Iron Steel grid on 


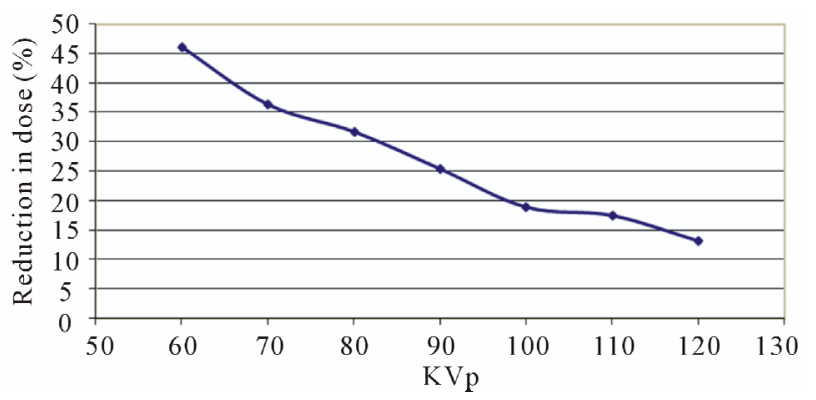

Figure 3. Percentage of reduction in backscattered X-ray dose.

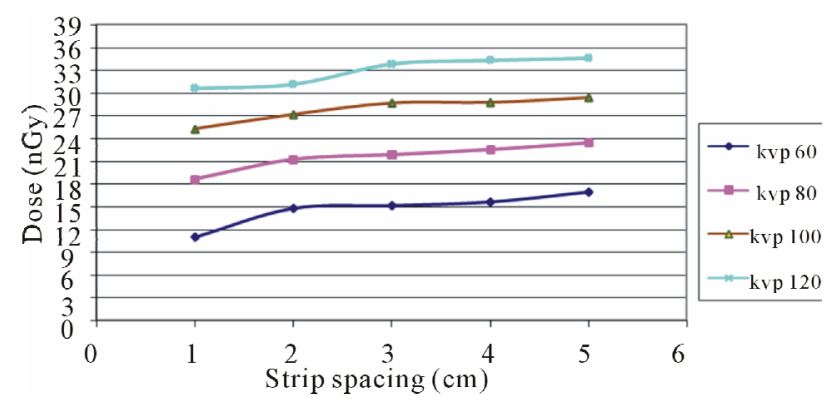

Figure 4. Backscattered radiation dose of X-ray at different strip spacing.

the reduction backscattered radiation. It is possible to reduce backscattered radiation up to about $46 \%$ by using iron steel grid. It can be observed that the higher reduction of backscattered radiation is at $60 \mathrm{kvp}$. This means a lot of backscattered radiations are absorbed by the base and strips of iron steel grid, and the minimum backscattered radiation dose records when we use the grid under the radiographic phantom at low applied voltage.

Also, the results show that the increase of the spacing between grid strips will cause a decrease on the effect of grid in reduction of backscattered radiation. In radiography, Iron steel grid proves to be very effective in backscattered radiation reduction. Therefore, the use of radiographic grids is best-known as the most significant and effective mean of eliminating scattered radiation.

\section{REFERENCES}

[1] K. Katalin, "The Clinical Significance of Diagnostic Modalities: X-Ray,” Department of Radiology, Semmelweis University, Budapest, 2012. http://oftakonyv.reak.bme.hu/tiki/index.php?

[2] O. Denise, “Scatter Control and Grid Use,” 2012. www.unisanet.unisa.edu.au/Resources/.../Scatter\%20Cont rol.ppt.2007

[3] Y. Jennifer, “Complex Problem Solving in Radiologic Technology: Understanding the Role of Experience Reflective Judgment and Workplace Culture,” Ph.D., Colombia University, New York, 2011.

[4] A. H. H. Mohamed, "Determination of Scattered Radiation to the Testis during Pa Chest X-Ray Procedures," Distance Education Project Report, Universiti Sains Malaysia, Pulau Pinang, 2007.

[5] S. Abdul-Majid, A. Kinsara, A. Almasoumi and M. Kallothody, "Reduction of Backscattered Radiation in Enclosure X-Ray Radiography,” 2005, pp. 27-30.

[6] W. Yao and K. Leszczynski, "Depth Distribution of Multiple Order X-Ray Scatter,” Radiation Physics and Chemistry, Vol. 77, No. 4, 2008, pp. 381-390. doi:10.1016/j.radphyschem.2007.12.002

[7] G. Wu, J. G. Mainprize, J. M. Boone and M. J. Yaffe, "Evaluation of Scatter Effects on Image Quality for Breast Tomosynthesis," Medical Physics, Vol. 36, No. 10, 2009, p. 4425. doi:10.1118/1.3215926 